\title{
QUANTIZATION OPERATORS AND INVARIANTS OF GROUP REPRESENTATIONS
}

\author{
ANDRÉS VIÑA
}

Communicated by Ivaïlo M. Mladenov

\begin{abstract}
Let $G$ be a semi-simple Lie group and $\pi$ some representation of $G$ belonging to the discrete series. We give interpretations of the constant $\pi(g)$, for $g \in Z(G)$, in terms of geometric concepts associated with the flag manifold $M$ of $G$. In particular, when $G$ is compact this constant is related to the action integral around closed curves in $M$. As a consequence, we obtain a lower bound for de cardinal of the fundamental group of $\operatorname{Ham}(M)$, the Hamiltonian group of $M$. We also interpret geometrically the values of the infinitesimal character of $\pi$ in terms of quantization operators.
\end{abstract}

\section{Introduction}

Given a Lie group $G$, its coadjoint action on the dual of the Lie algebra of $G$ gives rise to the coadjoint orbits. On the other hand, associated with $G$ we have the set of the irreducible unitary representations of $G$. Thus, the group $G$ has attached a set of "geometric objects", the coadjoint orbits, and a set of "algebraic objects", its irreducible unitary representations.

The study of the possible relations between the set of the coadjoint orbits of $G$ and the unitary dual of $G$, which is the space of the equivalence classes of unitary irreducible representations, is the aim of the Orbit Method.

The origin of the Orbit Method is the following theorem due to Kirillov [2]

Theorem 1 (Kirillov). Let $G$ be a nilpotent connected simply connected Lie group. Then the unitary dual of $G$ is in bijective correspondence with the space of the coadjoint orbits of $G$.

Furthermore, Kirillov gave interpretations of various facts relative to representation theory in terms of the geometry of the coadjoint orbits. For example, if $\mathcal{O}$ is the coadjoint orbit of the element $\mu$ in the dual of the Lie algebra of $G$ and $\pi$ is the 
corresponding representation in the above bijection, then the character $\chi_{\pi}$ of $\pi$ is given by an integral on the orbit $\mathcal{O}$

$$
\chi_{\pi}(\exp (A))=\int_{\mathcal{O}} \mathrm{e}^{2 \pi \mathrm{i} \mu(A)} \mathrm{d} \text { vol }
$$

for $A \in \mathfrak{g}:=\operatorname{Lie}(G)$. In Kirillov's exposition [4], this equality is called the sixth rule of the Orbit Method.

The Heisenberg group is nilpotent, connected and simply connected, and thus, it is an example to which Kirillov's theorem is applicable. The hypotheses of Kirillov's theorem are strong, and the assertion of this theorem is not true if we relax in a substantial way the hypotheses on the group $G$. For example, the complementary series of representations of $\mathrm{SL}(2, \mathbb{R})$ are not associated with coadjoint orbits.

The Orbit Method is based on the idea that a bijective map similar to the preceding one exists for any Lie group, if we modify the domain and the range of the map in an appropriate way [19-21]. Moreover, the Orbit Method tries to relate geometric properties of the coadjoint orbit with properties of the corresponding irreducible representation.

The "philosophical ground" of the Orbit Method is related with the quantization. Very roughly speaking, symplectic geometry is a mathematical model for classical mechanics. The phase space of a classical mechanical system is a symplectic manifold. A homogeneous $G$-manifold can be considered as a classical mechanical system equipped with a group of symmetries.

On the other hand, Hilbert spaces are reasonable mathematical models for quantum mechanics. Thus, a representation may be regarded as a quantum mechanical system endowed with a group of symmetries.

Classical and quantum mechanics can be considered as different descriptions of "the same physical system". So, for each classical system there should be a corresponding quantum system, and theoretically at least, one could construct from a classical system the respective quantum system. When there is the action of a group $G$, this construction, going from the orbit (the homogeneous $G$-space) to an irreducible representation is precisely what the Orbit Method asserts should exist.

The mathematical translation of this "philosophical" consideration is implemented by Geometric Quantization. Below we will recall briefly some points of this theory. Given a closed symplectic quantizable manifold $(N, \omega)$, there exists a complex line bundle $\mathcal{L}$ over $N$ (the prequantum bundle) whose first Chern class is essentially the cohomology class of $\omega$ [23].

Each Hamiltonian vector field $X$ on $N$ has associated a differential operator called quantization operator $\mathcal{Q}_{X}$, which acts on the sections of $\mathcal{L}$. These operators satisfy

$$
\mathcal{Q}_{[X, Y]}=\left[\mathcal{Q}_{X}, \mathcal{Q}_{Y}\right]
$$


where the bracket on the right hand side is for the commutator of the corresponding operators. If a Lie group $G$ acts on $N$ as a group of Hamiltonian symplectomorhisms, each $A \in \mathfrak{g}$ gives rise to a vector field $X_{A}$ on $M$ and the operators $\mathcal{Q}_{X_{A}}$ form a representation of the Lie algebra $\mathfrak{g}$.

When the group $G$ is compact and $N$ is a coadjoint orbit of an integral dominant element of $\mathfrak{g}^{*}$ endowed with the Kirillov symplectic structure, the corresponding prequantum bundle is $G$-equivariant. This property allows us to define a representation of $G$ on the space of sections of $\mathcal{L}$, in a natural way. The choice of a subalgebra of $\mathfrak{g}$ permits us to define polarized sections of $\mathcal{L}$. The restriction of the above representation to this space of the polarized sections is an irreducible representation of $G$. This is the Borel-Weil theorem regarded from the point of view of Geometric Quantization.

As we said, not every representation is associated to an orbit. In [17], we have studied the representations associated to hyperbolic orbits. Here we will consider a particular type of representations: the discrete series representations, whose definition is shortly reminded here.

Firstly, we will refer to the regular representation. For a Lie group $G$ the left regular representation is the space $L^{2}(G)$ endowed with the left translation. For $f \in L^{2}(G)$ and $g \in G, g \cdot f$ is defined by $(g \cdot f)(x)=f\left(g^{-1} x\right)$.

An irreducible unitary representation $\pi$ of a Lie group $G$ is said to be in the discrete series of $G$ if it can be realized as a direct summand of the left regular representation [1]. This is equivalent to the fact that the Plancherel measure for the decomposition of $L^{2}(G)$ assigns strictly positive mass to the one-point set $\{\pi\}$ in the unitary dual of $G$ (from this property comes the name "discrete" series). For $G$ compact, every irreducible representation of $G$ belongs to the discrete series.

If a group possesses discrete series representations, then it contains a compact Cartan subgroup. Kostant and Langlands conjectured the realization of the discrete series by the $L^{2}$-cohomology of holomorphic line bundles over the quotient of the group by the compact Cartan subgroup. This conjecture has been proved by Wilfried Schmid [12,13].

\section{Objectives of the Paper}

In the spirit of the Orbit Method and using the geometric construction of Schmid, we will describe here interpretations of some invariants of discrete series representations in terms of geometric concepts relative to the corresponding orbits.

Let $G$ be a linear semi-simple group, $T$ a compact Cartan subgroup of $G$ and $\pi$ a representation in the discrete series of $G$. We will consider the following points 
1) If $g_{1}$ belongs to the centre of $G$, then the corresponding operator $\pi\left(g_{1}\right)$ commutes with the operators $\pi(h)$ with $h \in G$. Schur's lemma asserts that $\pi\left(g_{1}\right)$ is a scalar operator, a multiple of the identity. That is, $\pi\left(g_{1}\right)$ is $\kappa$ times the identity. Thus, to each element in the centre of $G$ the representation $\pi$ assigns a complex constant $\kappa \in \mathrm{U}(1)$. We will give a geometric interpretation of the invariants $\kappa$ in terms of objects related with manifold $G / T$, which is an orbit of the $G$-action in the flag variety of the complexification $\mathfrak{g}_{\mathbb{C}}$ of $\mathfrak{g}$. When $G$ is compact, we will express $\kappa$ in terms of the symplectic action around closed curves in $G / T$. This is the first purpose of the paper.

2) On the other hand, the differential representation $\pi^{\prime}$ of $\pi$ is a representation of the Lie algebra of $G$, which gives rise to an irreducible representation of the universal enveloping algebra $U\left(\mathfrak{g}_{\mathbb{C}}\right)$ of $\mathfrak{g}_{\mathbb{C}}$. The simplest nontrivial invariant of $\pi^{\prime}$ is its infinitesimal character $\chi$, which gives the action of the center of $U\left(\mathfrak{g}_{\mathbb{C}}\right)$. The second purpose of this article is to express the infinitesimal character $\chi$ in terms of the geometry of $M=G / T$. More concretely, we relate the invariant $\chi$ with "quantization operators", which act on sections of a vector bundle over $G / T$.

3) Finally, we use the above results to give a lower bound for the cardinal of the homotopy group of the Hamiltonian group of the orbit $M$.

For a precise formulation of our results, we need to review briefly, following Schmid, the construction of the representation associated to an element $\phi$ in the weight lattice of $t$.

\section{Geometrical Framework}

Let $G$ be a linear semi-simple Lie group, $T$ a compact maximal Cartan subgroup and $K$ a maximal compact subgroup of $G$ containing $T$. By $\Delta$ we denote a positive root system of $\mathfrak{t}_{\mathbb{C}}=\mathfrak{t} \otimes \mathbb{C}$. We set

$$
\mathfrak{u}=\bigoplus_{\nu \in \Delta} \mathfrak{g}^{-\nu}, \quad \overline{\mathfrak{u}}=\bigoplus_{\nu \in \Delta} \mathfrak{g}^{\nu}
$$

$\mathfrak{g}^{\nu}$ being the root space of $\nu$. A root $\nu$ is said to be compact if the corresponding root space is contained in the Lie algebra $\mathfrak{k}_{\mathbb{C}}=\mathfrak{k} \otimes \mathbb{C}$. We will say that it is noncompact if $\mathfrak{g}^{\nu}$ is orthogonal to $\mathfrak{k}_{\mathbb{C}}$ with respect to the Killing form. We denote by $\mathfrak{b}$ the Borel subalgebra $\mathfrak{t}_{\mathbb{C}} \oplus \mathfrak{u}$.

As usual, $\rho$ stands for the half the sum of positive roots. Let $\phi$ be an element of the weight lattice of $\mathfrak{t}$. Denoting by $(.,$.$) the bilinear form on \mathfrak{t}^{*}$ induced by the Killing form, we put $q$ for the integer obtained as the sum of the cardinals of two 
sets of positive roots

$$
\begin{aligned}
q:= & \#\{\nu \in \Delta ; \nu \text { compact, }(\phi+\rho, \nu)<0\} \\
& +\#\{\nu \in \Delta ; \nu \text { noncompact, }(\phi+\rho, \nu)>0\} .
\end{aligned}
$$

In particular, when $G$ is compact and $\phi$ is a dominant weight, $q=0$.

By $B$ we denote the Borel subgroup of $G_{\mathbb{C}}$, determined by the $(T, \Delta)$. The $G$ orbit of $\mathfrak{b}$ in the flag manifold $G_{\mathbb{C}} / B$ is a complex submanifold that is isomorphic to $G / T$ and will be denoted by $M$. The weight $\phi$ induces a character $\Phi$ on $B$, which in turn allows us to define the holomorphic line bundle $\mathcal{V}=G_{\mathbb{C}} \times{ }_{B} \mathbb{C}$ over the flag manifold $G_{\mathbb{C}} / B$

$$
\mathcal{V}=\left\{(g, z) ; g \in G_{\mathbb{C}}, z \in \mathbb{C}\right\} / \sim
$$

where $(g, z) \sim\left(g b, \Phi\left(b^{-1}\right) z\right)$, with $b \in B$.

On the space of compactly supported $\mathcal{V}$-valued $(0, *)$-forms on $M$, we have the Dolbeault operator $\bar{\partial}: \mathcal{A}^{0, *}(\mathcal{V}) \rightarrow \mathcal{A}^{0, *+1}(\mathcal{V})$.

Furthermore, the group $G$ acts on the space $\mathcal{A}^{0, i}(\mathcal{V})$ by translation and the action commutes with the operator $\bar{\partial}$. By means of $G$-invariant Hermitian metrics on $M$ and on $\mathcal{V}$, we define the operator $\bar{\partial}^{*}$, the formal adjoint of $\bar{\partial}$. The space of square integrable, $C^{\infty}, \mathcal{V}$-valued $(0, q)$-forms on $M$ which belong to the intersection $\operatorname{ker}(\bar{\partial}) \cap \operatorname{ker}\left(\bar{\partial}^{*}\right)$ is denoted by $\mathcal{H}$.

According to the Langlands conjecture, if $\phi+\rho$ is regular, the action of $G$ on $\mathcal{H}$ is an irreducible unitary representation $\pi$, equivalent to a discrete series representation [1]. When $G$ is compact, the integer $q=0$, as we said, and $\mathcal{H}$ is precisely the representation given by Borel-Weil theorem.

Note that every $\sigma \in \mathcal{H}$ is, in fact, a $\bar{\partial}$-closed smooth Dolbeault form, but $\mathcal{H}$ can not be identified with the cohomology $H^{q}(M, \mathcal{O}(\mathcal{V}))$, since $M$ is not compact, in general.

Following the pattern of Geometric Quantization, it is convenient to consider the space $\mathcal{H}$ as a set of sections of an appropriate vector bundle. For this, we put $W:=\mathbb{C} \otimes\left(\bigwedge^{q} \mathfrak{u}\right)^{*}$ and we consider the representation $\Psi$ of $T$, tensor product of $\Phi$ by the $q$-exterior product of $\mathrm{Ad}^{*}$, i.e., $\Psi$ is a representation of $T$ on the vector space $W$. We denote by $\mathcal{P}$ the $\operatorname{GL}(W)$-principal bundle over $M$ determined by $\Psi$, and by $\mathcal{W}$ the associated vector bundle with fiber $W$ (defined by the standard representation of $\mathrm{GL}(W)$ ). That is

$$
\mathcal{P}=G \times_{\Psi} \mathrm{GL}(W) \rightarrow M=G / T
$$

and

$$
\mathcal{W}:=G \times_{\Psi} W=\{(g, v) \in G \times W\} / \sim
$$


with $(g, v) \simeq\left(g l, \Psi\left(l^{-1}\right) v\right)$ and $l \in T$. Thus, the vector space $\mathcal{H}$ is contained in the space $\Gamma(\mathcal{W})$ of sections of $\mathcal{W}$.

On $\mathcal{P}$ it is possible to define a $G$-invariant connection [18] and the corresponding covariant derivative in $\mathcal{W}$ will be denoted $\nabla$.

The bundles $\mathcal{P}$ and $\mathcal{W}$ will be the geometric framework for developments presented below. The vector bundle $\mathcal{W}$ will play a similar role as the prequantum bundle in Geometric Quantization.

Taking into account the natural actions of $G$ on $\mathcal{W}$ and on $G / T$, one can define the following representation of $G$ on the space $\Gamma(\mathcal{W})$

$$
(g \cdot \sigma)(x)=g\left(\sigma\left(g^{-1} x\right)\right)
$$

for $\sigma \in \Gamma(\mathcal{W})$ and $x \in M=G / T$. Its restriction to the subspace $\mathcal{H}$ of $\Gamma(\mathcal{W})$ is the discrete series representation $\pi$.

The space of sections $\Gamma(\mathcal{W})$ can be identified with the space of $\Psi$-equivariant functions, i.e., functions $s: G \rightarrow W$ satisfying $s(g l)=\Psi\left(l^{-1}\right) s(g)$, for all $l \in T$ and all $g \in G$. A section $\sigma$ is related with a $\Psi$-equivariant function $s$ by the formula $\sigma(g T)=\langle g, s(g)\rangle$, where $\langle g, v\rangle$ denotes the element in $\mathcal{W}$ determined by the pair $(g, v) \in G \times W$. It easy to prove that the representation $\pi$, in terms of $\Psi$-equivariant functions, is given by

$$
\pi(g)(s)=s \circ L_{g^{-1}}
$$

where $L$ is the left translation in $G$.

\section{Differential Representation and Quantization Operators}

Throughout, we will assume that the element $\phi+\rho \in$ it $^{*}$ is regular. We will denote by $\mathcal{H}_{K}$ the space of $K$-finite vectors of $\mathcal{H}$, namely vectors in $\mathcal{H}$ which belong to a finite-dimensional $K$-invariant subspace. In other words, $\mathcal{H}_{K}$ is the Harish-Chandra module of $\mathcal{H}$ [14]. The differential representation, on the space $\mathcal{H}_{K}$, of the above irreducible unitary representation $\pi$ will be denoted by $\pi^{\prime}$.

The decomposition of $\mathfrak{g}_{\mathbb{C}}$ as direct sum of root spaces

$$
\mathfrak{g}_{\mathbb{C}}=\mathfrak{t}_{\mathbb{C}} \oplus \bigoplus_{\nu \in \Delta}\left(\mathfrak{g}^{\nu} \oplus \mathfrak{g}^{-\nu}\right)
$$

induces a direct sum decomposition for $\mathfrak{g}$ of the form

$$
\mathfrak{g}=\mathfrak{t} \oplus \mathfrak{l} .
$$


The projection of an element $C$ of $\mathfrak{g}$ on $\mathfrak{t}$ will be denoted $C_{0}$. Thus, given $A \in \mathfrak{g}$, we can define

$$
h_{A}: G \rightarrow \mathfrak{g l}(W) \quad \text { with } \quad h_{A}(g)=\Psi^{\prime}\left(\left(g^{-1} \cdot A\right)_{0}\right)
$$

where the dot means adjoint action. By means of $h_{A}$ we define the endomorphism $F_{A}$ of the fibre bundle $\mathcal{W}$

$$
F_{A}: \mathcal{W} \rightarrow \mathcal{W} \quad \text { with } \quad F_{A}(\langle g, v\rangle)=\left\langle g, h_{A}(g)(v)\right\rangle .
$$

For $A \in \mathfrak{g}$, we denote by $X_{A}$ the vector field on $M$ determined by $A$, and we define the following first order differential operator, which acts on the space of sections $\Gamma(\mathcal{W})$

$$
\mathcal{Q}_{A}:=-\nabla_{X_{A}}+F_{A} .
$$

When $G$ is a compact group and $\phi$ is a dominant weight, then $q=0, \mathcal{W}$ is a prequantum bundle and $\mathcal{Q}_{A}$ is the respective quantization operator associated with the vector field $X_{A}$ in Geometric Quantization [15].

By abusing of language, the operators $\mathcal{Q}_{A}$ will be called "quantization operators", although $G$ is not necessarily compact. The following theorem expresses the differential representation $\pi^{\prime}$ as an action on geometric objects (see [17,18] for proof)

Theorem 2. The map $A \mapsto \mathcal{Q}_{A}$ defines a representation of the Lie algebra $\mathfrak{g}$ on the space $\mathcal{H}_{K}$, which is equivalent to $\pi^{\prime}$.

The universal enveloping algebra $U\left(\mathfrak{g}_{\mathbb{C}}\right)$ is defined as the quotient of the tensor algebra $\mathcal{T}\left(\mathfrak{g}_{\mathbb{C}}\right)$ by the two-sided ideal generated by the set

$$
\left\{X \otimes Y-Y \otimes X-[X, Y] ; X, Y \in \mathfrak{g}_{\mathbb{C}}\right\} .
$$

Each representation of $\mathfrak{g}$, in particular $\pi^{\prime}$, determines a representation of the associative algebra $\mathrm{U}\left(\mathfrak{g}_{\mathbb{C}}\right)$. The elements of the centre of the universal enveloping algebra play an important role in representation theory (among the elements of degree two in the centre is the Casimir element) $[5,6]$. As a consequence of the generalization of Schur's lemma due to Dixmier, it turns out that the centre $\mathcal{Z}\left(\mathfrak{g}_{\mathbb{C}}\right)$ of $U\left(\mathfrak{g}_{\mathbb{C}}\right)$ acts by scalars in the representation induced by $\pi^{\prime}$. The resulting homomorphism

$$
\chi: \mathcal{Z}\left(\mathfrak{g}_{\mathbb{C}}\right) \rightarrow \mathbb{C}
$$

is called an infinitesimal character of the $\mathrm{U}\left(\mathfrak{g}_{\mathbb{C}}\right)$-module $\mathcal{H}_{K}$.

Let $\left\{C_{1}, \ldots, C_{r}\right\}$ be a basis of $\mathfrak{t}_{\mathbb{C}}$ and $E_{\nu}$ a basis of $\mathfrak{g}^{\nu}$. Each element $J \in \mathcal{Z}\left(\mathfrak{g}_{\mathbb{C}}\right)$ is a polynomial $p\left(C_{i}, E_{\nu}\right)$ in the variables $C_{i}$ and $E_{\nu}$. One can prove the following theorem $[17,18]$. 
Theorem 3. The differential operator $p\left(\mathcal{Q}_{C_{i}}, \mathcal{Q}_{E_{\nu}}\right)$, obtained via substituting in the polynomial $p$ the corresponding "quantization operators", is the scalar one defined by the constant $\chi(J)$, which acts on the space $\mathcal{H}_{K}$.

\section{Invariants Defined by Schur's Lemma}

Given an element $g_{1}$ in the center of $G$, by Schur's lemma it is known that

$$
\pi\left(g_{1}\right)=\kappa \operatorname{Id}_{\mathcal{H}}
$$

$\kappa$ being a complex number of modulus one.

To know the action of $\pi\left(g_{1}\right)$, we will integrate $\pi^{\prime}$ along a curve in $G$ with initial point at the identity element and final point at $g_{1}$. In this way, we will give two geometric interpretations of the invariant $\kappa$ associated with $\pi$

i) in terms of "evolution equations" for elements in $\mathcal{H}$, equations generated by families of "quantization operators"

ii) as a gauge transformation of the fibre bundle $\mathcal{P}$, which is the time-one map of a flow in $\mathcal{P}$ that preserves the connection.

For the explanation of i) and ii) we need to introduce some notations. Henceforth, $\left\{g_{t} ; t \in[0,1]\right\}$ stands for a smooth curve in $G$ with the initial point at $e$. For brevity, such a curve will be called a path in $G$.

Given the path $\left\{g_{t}\right\}$, we denote by $\left\{A_{t}\right\} \subset \mathfrak{g}$ the corresponding velocity curve, that is,

$$
A_{t}=\dot{g}_{t} g_{t}^{-1}
$$

which in turn determines the "quantization operators" $\mathcal{Q}_{A_{t}}$. Furthermore, through the $G$-action on $M$, the path $g_{t}$ defines an isotopy of $M=G / T$, which will be denoted by $\left\{\varphi_{t}\right\}_{t \in[0,1]}$, i.e.,

$$
\varphi_{t}(g T)=g_{t} g T .
$$

Let $\left\{g_{t}\right\}$ be a path in $G$ and $\sigma$ a section of $\mathcal{W}$, we can consider the set of sections $\sigma_{t}$ of $\mathcal{W}$ determined by the following "evolution equations"

$$
\frac{\mathrm{d} \sigma_{t}}{\mathrm{~d} t}=\mathcal{Q}_{A_{t}}\left(\sigma_{t}\right), \quad \sigma_{0}=\sigma .
$$

When $g_{1}$ belongs to $Z(G)$, the center of $G$, the family $\left\{\varphi_{t}\right\}$ is a loop of diffeomorphisms of $M$, and one has the following theorem $[17,18]$ 
Theorem 4. If $g_{1} \in Z(G)$, then $\sigma_{1}=\kappa \sigma$, for any $\sigma \in \mathcal{H}$.

That is, in the evolution defined by (2), the section $\sigma$ takes at the instant $t=1$ the initial value multiplied by $\kappa$.

On the other hand, for each $A \in \mathfrak{g}$, the natural left $G$-action on the principal bundle $\mathcal{P}$ determines a vector field $Y_{A}$ on $\mathcal{P}$. Hence, a path $\left\{g_{t}\right\}$ in $G$ defines the time-dependent vector field $Y_{A_{t}}$, which in turn determines a flow $\mathbf{H}_{t}$ on $\mathcal{P}$. The following theorem gives other interpretation of $\kappa$, now in the context of the principal bundle $\mathcal{P}[17,18]$.

Theorem 5. If $g_{1} \in Z(G)$, the time-one map $\mathbf{H}_{1}$ of the flow $\mathbf{H}_{t}$ is the gauge transformation on $\mathcal{P}$ given by $\mathbf{H}_{1}(p)=p \kappa$.

That is, the flow along the integral curves of $Y_{A_{t}}$ moves a given point in such a way that at the instant $t=1$, the transported point reaches the initial fibre in a position that is the initial one multiplied by $\kappa$.

The constant $\kappa$ also appears in the evolution of $W$-valued GL( $W)$-equivariant functions on $\mathcal{P}$, as the following theorem shows $[17,18]$

Theorem 6. If $f_{t}: \mathcal{P} \rightarrow W$ is the family of $\mathrm{GL}(W)$-equivariant maps solutions of

$$
\frac{\mathrm{d} f_{t}}{\mathrm{~d} t}=-Y_{A_{t}}\left(f_{t}\right)
$$

with $f_{0}=f$ (an arbitrary $\mathrm{GL}(W)$-equivariant map), then $f_{1}=\kappa f$.

When $G$ is compact and $\phi$ is a regular dominant weight, the representation $\pi$ is the one provided by the Borel-Weil Theorem. In this case $M$ is the flag variety of $\mathfrak{g}_{\mathbb{C}}$, a compact manifold diffeomorphic to the coadjoint orbit of $\phi \in \mathfrak{g}^{*}$. On $M$ we will consider the symplectic Kirillov structure $\varpi$ [3]. If $\left\{g_{t}\right\}$ is a path with $g_{1} \in Z(G)$, then the respective isotopy $\left\{\varphi_{t}\right\}$ is a loop in $\operatorname{Ham}(M, \varpi)$, the Hamiltonian group [10] of $(M, \varpi)$, with $h_{A_{t}}$ the corresponding time-dependent Hamiltonian. Given an arbitrary point $x_{0} \in M$, the closed curve $\left\{\varphi_{t}\left(x_{0}\right)\right\}_{t}$, obtained by evaluation of the loop $\varphi_{t}$ at the point $x_{0}$, is nullhomologous [8]. The symplectic action around the loop $\left\{\varphi_{t}\right\}$ is the element of $\mathbb{R} / \mathbb{Z}$ defined by

$$
\mathcal{S} \mathcal{A}(\varphi):=\int_{S} \varpi+\int_{0}^{1} h_{A_{t}}\left(\varphi_{t}\left(x_{0}\right)\right) \mathrm{d} t
$$

$S$ being a two-chain whose boundary is $\left\{\varphi_{t}\left(x_{0}\right)\right\}_{t}[16,22]$.

From Theorem 3, one can deduce the following result $[17,18]$ 
Theorem 7. If $G$ is compact, $\phi$ is a regular dominant weight and $g_{1} \in Z(G)$, then $\kappa$ is the exponential of the symplectic action around the Hamiltonian loop $\varphi_{t}$. That is

$$
\kappa=\exp (\mathcal{S A}(\varphi))
$$

\section{Lower Bounds for the Cardinal of the Fundamental Group of Some Subgroups of $\operatorname{Diff}(M)$}

We will consider subalgebras $\mathfrak{X}$ of $\mathfrak{X}(M)$ (the Lie algebra consisting of the vector fields on $M$ ), such that its elements admit lifts to $\mathrm{GL}(W)$-invariant vector fields on $\mathcal{P}$ that are infinitesimal symmetries of the connection. The precise properties which characterize these algebras are written below (see [18] for more detailed explanations).

By $\mathfrak{X}$ we denote any Lie subalgebra of $\mathfrak{X}(M)$ satisfying the following conditions:

1) There is a continuous $\mathbb{R}$-linear map

$$
Z \in \mathfrak{X} \mapsto U(Z) \in \mathfrak{X}(\mathcal{P})
$$

such that $Z^{\natural}$, the horizontal lift of $Z$, is the horizontal component of $U(Z)$.

2) For each $Z \in \mathfrak{X}$, there is a $C^{\infty}$ map $a(Z): \mathcal{P} \rightarrow \mathfrak{g l}(W)$ such that

2a) $a(Z)(p \beta)=\beta^{-1} a(Z)(p) \beta$, for all $\beta \in \mathrm{GL}(W), p \in \mathcal{P}$. That is, $a(Z)$ is a pseudo-tensorial function on $\mathcal{P}$ of type $\mathrm{Ad}$ [7].

2b) $U(Z)=Z^{\natural}+V_{a(Z)}$, where $V_{a(Z)}$ is the vertical vector field on $\mathcal{P}$ defined by $a(Z)$.

2c) $D a(Z)=-\mathbf{K}\left(Z^{\natural},.\right)$, where $D$ is the covariant derivative and $\mathbf{K}$ is the curvature of the connection on $\mathcal{P}$.

2d) The map $Z \mapsto a(Z)$ is $\mathbb{R}$-linear and continuous.

Example 8. For $X_{C}$, with $C \in \mathfrak{g}$ we define $U\left(X_{C}\right):=Y_{C}$. We denote by $[g, \alpha]$ the element of $\mathcal{P}$ determined by $(g, \alpha) \in G \times \mathrm{GL}(W)$, and set

$$
a\left(X_{C}\right)([g, \alpha]):=\alpha^{-1} h_{C}(g) \alpha
$$

where $h_{C}$ is the map defined in 1). The Lie algebra $\mathfrak{X}:=\left\{X_{C} ; C \in \mathfrak{g}\right\}$, with these choices for $U\left(X_{C}\right)$ and $a\left(X_{C}\right)$, satisfies the conditions 1)-2d). 
Let $\left\{Z_{t}\right\}_{t \in[0,1]}$ be a time-dependent vector field on $M$, with $Z_{t} \in \mathfrak{X}$. Let $\left\{\psi_{t}\right\}$ be the isotopy of $M$ defined by

$$
\frac{\mathrm{d} \psi_{t}}{\mathrm{~d} t}=Z_{t} \circ \psi_{t}, \quad \psi_{0}=\operatorname{Id}_{M} .
$$

We have the time-dependent vector field $U_{t}:=U\left(Z_{t}\right)$ on $\mathcal{P}$ and the corresponding flow $\mathbf{H}_{t}$

$$
\frac{\mathrm{d} \mathbf{H}_{t}}{\mathrm{~d} t}=U_{t} \circ \mathbf{H}_{t}, \quad \mathbf{H}_{0}=\mathrm{Id}_{\mathcal{P}} .
$$

It is not hard to prove that $\mathbf{H}_{t}$ is a diffeomorphism of $\mathcal{P}$ over $\psi_{t}$ which preserves the connection (see [18]).

In particular, let $\left\{g_{t}\right\}$ be a path in $G$ and $\left\{A_{t}\right\}$ the corresponding velocity curve, such that $X_{A_{t}} \in \mathfrak{X}$. This family of vector fields gives rise to the time-dependent vector field on $\mathcal{P}, U\left(X_{A_{t}}\right)=Y_{A_{t}}$, which in turn defines a flow $\mathbf{H}_{t}$ on $\mathcal{P}$. If $g_{1}$ is an element of the center of $G$, then $\left\{\varphi_{t}\right\}$ is a loop and $\mathbf{H}_{1}$ is a gauge transformation of $\mathcal{P}$. Furthermore, by Theorem 5, for all $p \in \mathcal{P}, \mathbf{H}_{1}(p)=p \kappa$, with $\kappa=\Phi\left(g_{1}\right)$.

We can consider the $T$-principal bundle $T \rightarrow G \rightarrow M=G / T$, endowed with the invariant connection [7, page 103] determined by the splitting $\mathfrak{g}=\mathfrak{t} \oplus \mathfrak{l}$ introduced in Section 3. For each $g_{1} \in Z(G)$, there is a path in $G$ which is horizontal with respect to this connection and with endpoint at $g_{1}$ (see [18]). These particular paths will be considered in the following paragraph.

Let $\mathcal{G}$ be a connected Lie subgroup of $\operatorname{Diff}(M)$ such that, it contains the isotopies associated with paths in $G$, and $\operatorname{Lie}(\mathcal{G})$ is subalgebra of some algebra $\mathfrak{X}$. Let $g_{t}$ and $\tilde{g}_{t}$ be paths in $G$ with final point in $Z(G)$ and horizontal with respect the $T$ invariant connection above mentioned. These paths define the loops $\varphi$ and $\tilde{\varphi}$ in the group $\mathcal{G}$, respectively. Let $\left\{\xi^{s}\right\}_{s}$ be a homotopy in $\mathcal{G}$ between the loops $\varphi$ and $\tilde{\varphi}$. Using properties of the bundle isomorphisms of $\mathcal{P} \mathbf{H}_{t}, \tilde{\mathbf{H}}_{t}$ and $\mathbf{H}_{t}^{s}$, (determined $\varphi, \tilde{\varphi}$ and $\xi^{s}$, respectively) one can prove that $\Phi\left(g_{1}\right)=\Phi\left(\tilde{g}_{1}\right)$. In other words, we have the following theorem (see [18])

Theorem 9. Let $\mathcal{G}$ be a connected Lie subgroup of $\operatorname{Diff}(M)$, such that

i) it contains the isotopies associated with paths in $G$.

ii) Lie $(\mathcal{G})$ is subalgebra of some algebra $\mathfrak{X}$.

Then

$$
\#\left(\pi_{1}(\mathcal{G})\right) \geq \#\{\Phi(g) ; g \in Z(G)\} .
$$


If $G$ is compact, $\phi$ is a regular dominant weight and $\mathcal{G}$ is any subgroup of the Hamiltonian group $\operatorname{Ham}(M, \varpi)$, then $\operatorname{Lie}(\mathcal{G})$ satisfies condition ii) in the statement of theorem (see [18]). Thus, we have the following corollary

Corollary 10. If $G$ is compact, $\phi$ is a regular dominant weight and $\mathcal{G}$ is any connected subgroup of $\operatorname{Ham}(M, \varpi)$ that contains $G$, then

$$
\#\{\Phi(g) ; g \in Z(G)\}
$$

is a lower bound of $\#\left(\pi_{1}(\mathcal{G})\right)$.

Example 11. For $G=\mathrm{SU}(2)$, the corresponding flag manifold is $\mathbb{C P}^{1}$. On the other hand, let $\phi$ be the weight of $T=\mathrm{U}(1)$ defined on $\mathrm{t}$ by

$$
\phi(\operatorname{diag}(a \mathrm{i},-a \mathrm{i}))=a .
$$

The corresponding Kirillov symplectic structure $\varpi$ is equal to $-2 \pi \omega_{F S}$, where $\omega_{F S}$ is de Fubini-Study symplectic form. Thus

$$
\operatorname{Ham}\left(\mathbb{C P}^{1}, \varpi\right) \simeq \operatorname{Ham}\left(\mathbb{C P}^{1}, \omega_{F S}\right) .
$$

By Corollary 10

$$
\#\left\{\pi_{1}\left(\operatorname{Ham}\left(\mathbb{C P}^{1}, \varpi\right)\right)\right\} \geq 2 .
$$

The homotopy type of the Hamiltonian groups $\operatorname{Ham}(N, \Omega)$ is known only for some symplectic manifolds [9]. However, for the simplest case of $\mathbb{C P}^{1}$ endowed with the Fubini-Study form, one has see page 52 of [11]

$$
\pi_{1}\left(\operatorname{Ham}\left(\mathbb{C P}^{1}, \omega_{F S}\right)\right) \simeq \mathbb{Z} / 2 \mathbb{Z} .
$$

Thus, in this particular case, the lower bound given in Corollary 10 is precisely the cardinal of the fundamental group of the Hamiltonian group.

\section{Acknowledgements}

This work has been partially supported by Ministerio de Ciencia y Tecnología, grant FPA2009-11061.

\section{References}

[1] Bolton V. and Schmid W., Discrete Series, In: Proc. of Symposia in Pure Math. 61 (1997) 83-113. 
[2] Kirillov A., Unitary Representations of Nilpotent Lie Groups, Uspehi Mat. Nauk. 17 (1962) 57-110.

[3] Kirillov A., Elements of the Theory of Representations, Springer, Berlin 1976.

[4] Kirillov A., Lectures on the Orbit Method, American Mathematical Society, Providence 2004.

[5] Knapp A., Representation Theory of Semi-simple Groups: An Overwiew Based on Examples, Princeton University Press, Princeton 2001.

[6] Knapp A., Lie Groups Beyond an Introduction, Progr. Math. vol. 140, Birkhäuser, Boston 2005.

[7] Kobayashi S. and Nomizu K., Foundations of Differential Geometry vol I, Interscience, New York 1963.

[8] Lalonde F., McDuff D. and Polterovich L., On Flux Conjectures. In: CRM Proceedings and Lecture Notes 15, AMS, Providence 1998, pp. 69-85.

[9] McDuff D., A Survey of the Topological Properties of Symplectomorphism Groups, In: Topology, Geometry and Quantum Field Theory, London Math. Soc. Lecture Note Ser. vol. 308, Cambridge U. P., Cambridge 2004, pp. 173193.

[10] McDuff D. and Salamon D., Introduction to Symplectic Topology, Clarendon Press, Oxford 1998.

[11] Polterovich L., The Geometry of the Group of Symplectic Diffeomorphisms, Birkhäuser, Basel 2001.

[12] Schmid W., On a Conjeture of Langlands, Ann. Math. 93 (1971) 1-42.

[13] Schmid W., $L^{2}$-Cohomology and the Discrete Series, Ann. Math. 103 (1976) 375-394.

[14] Schmid W., Geometric Methods in Representation Theory, In: Poisson Geometry, Deformation Quantisation and Group Representations, S. Gutt, J. Rawnsley and D. Sternheimer (Eds), London Mathematical Society, Lecture Note Series vol. 323, Cambridge U.P., Cambridge 2005, pp. 273-324.

[15] Śniatycki J., Geometric Quantization and Quantum Mechanics, Springer, New York 1980.

[16] Viña A., Symplectic Action around Loops in $\operatorname{Ham}(M)$, Geom. Dedicata 109 (2004) 31-49.

[17] Viña A., Action Integrals and Infinitesimal Characters, Lett. Math. Phys. 91 (2010) 241-264.

[18] Viña A., Action Integrals and Discrete Series, arXiv:1108.1611 [math.SG] 
[19] Vogan D., Unitary Representations of Reductive Lie Groups, Princeton University Press, Princeton 1987.

[20] Vogan D., The Orbit Method and Unitary Representations, Algebraic and Analytic Methods in Representation Theory (Sonderborg, 1994), In: Perspectives in Mathematics vol. 17, Academic Press, San Diego 1997, pp 243-339.

[21] Vogan D., The Orbit Method of Coadjoint Orbits for Real Reductive Groups. In: Representation Theory of Lie Groups, J. Adams and D. Vogan (Eds), IAS/Park City Mathematics Series vol. 8, AMS, Providence 1999, pp. 177238.

[22] Weinstein A., Cohomology of Symplectomorphism Groups and Critical Values of Hamiltonians, Math. Z. 201 (1989) 75-82.

[23] Woodhouse N., Geometric Quantization, Clarendon Press, Oxford 1992.

Andrés Viña

Departamento de Física

Universidad de Oviedo

Avda Calvo Sotelo

33007 Oviedo, SPAIN

E-mail address: vina@uniovi.es 